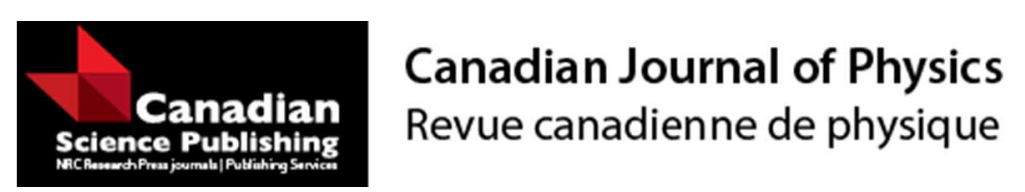

\title{
A general formula for calculation of the two-dimensional Franck-Condon Factors
}

\begin{tabular}{|c|c|}
\hline Journal: & Canadian Journal of Physics \\
\hline Manuscript ID & cjp-2016-0475.R2 \\
\hline Manuscript Type: & Article \\
\hline Date Submitted by the Author: & 13-Dec-2016 \\
\hline Complete List of Authors: & $\begin{array}{l}\text { Koc, Huseyin; Mus Alparslan Universitesi, } \\
\text { Mamedov, Bahtiyar; University } \\
\text { Eser, Erhan; Gazi Universitesi }\end{array}$ \\
\hline Keyword: & $\begin{array}{l}\text { Franck-Condon integral, Duschinsky effects, Binomial coefficients, Hermite } \\
\text { polynomials, Harmonic oscillators }\end{array}$ \\
\hline
\end{tabular}

\section{SCHOLARONE ${ }^{\text {m }}$}

Manuscripts 


\section{A general formula for calculation of the two-dimensional Franck-Condon Factors}

H. Koç ${ }^{a}$, B. A. Mamedov ${ }^{b}$, and E. Eser ${ }^{c}$

${ }^{a}$ Department of Electrical and Electronics Engineering, Faculty of Engineering, Muş Alparslan University, Muş, Turkey

${ }^{\mathrm{b}}$ Department of Physics, Faculty of Arts and Sciences, Gaziosmanpaşa University, Tokat, Turkey

${ }^{c}$ Department of Physics, Polatlı Faculty of Arts and Sciences, Gazi University, Polatl1, Ankara, Turkey

Corresponding author: huseyinnkoc@yahoo.com

Address: Department of Electrical and Electronics Engineering, Faculty of Engineering, Muş Alparslan University, 49250- Muş, Turkey

Telefon: +90 (436) 2491081

Fax $\quad$ : +90(436) 2120853

PACS Number: 33.70.Ca, 03.65.Fd, 31.15.xr, 31.15.-p 


\title{
A general formula for calculation of the two-dimensional Franck-Condon
}

\section{Factors}

\begin{abstract}
Knowledge of the FC factors and related quantities is essential to understand and to estimate many important aspects of the astrophysical molecules, such as kinetics of the energy transfer, radiative life times, band intensity and vibrational temperatures. In this view, we propose a new analytical formula of the Franck-Condon (FC) integral for two-dimensional harmonic oscillators taking into account the Duschinsky effect. This method is based on the use of binomial expansion theorem and the Hermite polynomials. With the formula obtained, the FCF of any transition can be computed independently. In this study, the method for FCF calculations was applied to $\mathrm{NO}_{2}$ molecule.
\end{abstract}

Keywords: Franck-Condon Factor, Duschinsky effects, Binomial coefficients, Hermite polynomials

\section{Introduction}

Squares of the Franck-Condon (FC) overlap integrals are the so-called FC factor, which is a fundamental method in molecular physics [1-16]. The FC principle determines not only the transition probabilities between different vibrational levels of the two electronic states, but also the spectral line intensities and the transition rates of diatomic and polyatomic molecules [17-21]. For diagnostic applications in astronomy, astrophysics and allied subjects, the transition probability, which is proportional with the FC factor, parameters are required [22]. Accurate evaluation of the multidimensional FC factor including Duschinsky effects is therefore of primary importance. The Duschinsky effect arises in the coordinate transformations between the normal vibrational motions of the ground and first excited state [23]. The FC factor including Duschinsky effects has been studied for a long time, both theoretically and experimentally, for the solution of many problems $[10,11,24]$, and in this 
manner many new analytical formulae of FCI for multidimensional harmonic oscillators have been developed [3, 12, 14, 24-27]. The studies generally have been made on the basis of the harmonic oscillator wave function [16], and there also are the FCF calculations including anhorminicity by simulation $[8,9,28,29]$. The obtained analytical expressions are tested to investigate the photoelectron spectra of various molecules.

Analytical expressions of the multidimensional FC integrals (FCI) for the polyatomic molecules can be formed by obtaining the multivariable functions. These functions include the frequency variations and the coordinate transformations between the excited states and the ground state [1].

The aim of this study is to derive a simple and easily computable analytical formula for FCI on the basis of the two-dimensional harmonic oscillator wave function.

\section{The Two-dimensional Franck-Condon Factors}

The normalized vibrational overlap integral over n-dimensional harmonic oscillators wave functions centered at different equilibrium positions is given by [13, 24]

$$
\left\langle v_{1} \ldots v_{n} \mid v_{1}^{\prime} \ldots v_{n}^{\prime}\right\rangle=N \int_{-\infty}^{\infty} \ldots \int_{-\infty}^{\infty} \psi_{v_{1} \ldots v_{n}}\left(Q_{1} \ldots Q_{n}\right) \psi_{v_{1}^{\prime} \ldots v_{n}^{\prime}}\left(Q_{1}^{\prime} \ldots Q_{n}^{\prime}\right) d Q_{1} d Q_{2} \ldots d Q_{n}
$$

Where $N=\operatorname{det}(J)^{1 / 2}$ is normalization constant, $v_{1}, v_{2}, \cdots v_{n}$ are the vibrational quantum numbers and $\psi_{v_{1} \ldots v_{n}}\left(Q_{1} \ldots Q_{n}\right)$ are the n-dimensional oscillators wave functions defined as

$$
\psi_{v_{1} \ldots v_{n}}\left(Q_{1} \ldots Q_{n}\right)=\prod_{j=1}^{N} N_{j} H_{v_{j}}\left(\gamma_{j}^{1 / 2} Q_{j}\right) \exp \left(-\gamma_{j} Q_{j}^{2} / 2\right)
$$


Here, $\gamma_{j}=\omega_{j} / \hbar$ is related to the frequency $\left(w_{j}\right.$ is jth mode angular frequency, $\hbar$ is Planck's constant), $N_{j}=\left(\sqrt{\gamma_{j} / \pi} / 2^{v_{j}} v_{j} !\right)^{1 / 2}$ is normalization constant, and the Hermite polynomials defined as [16]

$$
H_{n}(x)=\frac{2^{n-1}}{\sqrt{\pi}} \sum_{k=0}^{n} f_{k}(n) x^{n-k} i^{k} \Gamma\left(\frac{1+k}{2}\right)\left[1+(-1)^{k}\right],
$$

where $f_{m}(n)=n ! /[m !(n-m) !]$ is the binomial coefficients and $i=\sqrt{-1}$.

The aim of this study is to obtain the analytical formulae for the two-dimensional FCI over harmonic oscillator wave functions. In this manner, in the special case, the two-dimensional FCI of harmonic oscillators (Eq. (1)) can be written as [26]

$$
\begin{aligned}
\left\langle n_{1} m_{1} \mid n_{2} m_{2}\right\rangle=N \int_{-\infty}^{\infty} \int_{-\infty}^{\infty} H_{n_{1}}\left(\sqrt{\alpha_{1}} x_{1}\right) H_{m_{1}}\left(\sqrt{\alpha_{2}} x_{2}\right) H_{n_{2}}\left(\sqrt{\beta_{1}} x_{1}^{\prime}\right) H_{m_{2}}\left(\sqrt{\beta_{2}} x_{2}^{\prime}\right) \\
\times \exp \left[-\frac{1}{2}\left(\alpha_{1} x_{1}^{2}+\alpha_{2} x_{2}^{2}+\beta_{1} x_{1}^{\prime 2}+\beta_{2} x_{2}^{\prime 2}\right)\right] d x_{1} d x_{2}
\end{aligned}
$$

where

$$
N=\frac{1}{\pi} \prod_{i=1}^{2}\left(\frac{\sqrt{\alpha_{i} \beta_{i}}}{2^{n_{i}+m_{i}} n_{i} ! m_{i} !}\right)^{1 / 2}
$$

and

$$
\left(\begin{array}{c}
x_{1}^{\prime} \\
x_{2}^{\prime}
\end{array}\right)=\left(\begin{array}{ll}
J_{11} & J_{12} \\
J_{21} & J_{22}
\end{array}\right)\left(\begin{array}{l}
x_{1} \\
x_{2}
\end{array}\right)+\left(\begin{array}{l}
d_{1} \\
d_{2}
\end{array}\right) \text {. }
$$

Here $J_{i j}$ is the element of the Duschinsky matrix and $d_{i}$ is the geometrical difference [26,]. Lee et al. [26] proposed an efficient method for the calculation of the two-dimensional FCI using the Duschinsky effect. 


$$
\begin{array}{r}
\left\langle n_{1} m_{1} \mid n_{2} m_{2}\right\rangle=N \int_{-\infty}^{\infty} \int_{-\infty}^{\infty} H_{n_{1}}\left(a_{1}\right) H_{m_{1}}\left(a_{2}\right) H_{n_{2}}\left(a_{3}\right) H_{m_{2}}\left(a_{4}\right) \\
\times \exp \left[-\frac{1}{2}\left(a_{3}^{2}+a_{4}^{4}+a_{5}\right)\right] d x_{1} d x_{2}
\end{array}
$$

Here, the coefficients of Hermite polynomials are as follows:

$$
\begin{aligned}
& a_{1}=\sqrt{\alpha_{1}} x_{1} \\
& a_{2}=\sqrt{\alpha_{2}} x_{2} \\
& a_{3}=\sqrt{\beta_{1}}\left(\sum_{i=1}^{2} J_{1 i} x_{i}+d_{1}\right) \\
& a_{4}=\sqrt{\beta_{2}}\left(\sum_{i=1}^{2} J_{2 i} x_{i}+d_{2}\right) \\
& a_{5}=\sum_{i=1}^{2} \alpha_{i} x_{i}^{2}
\end{aligned}
$$

Substituting Eq. (3) into Eq. (7), we can obtain the formulae

$$
\left\langle n_{1} m_{1} \mid n_{2} m_{2}\right\rangle=N \cdot K_{n_{1} m_{1}}^{n_{2} m_{2}} \cdot I_{n_{1} m_{1} ; a_{1} a_{2} a_{3}}^{n_{2} m_{2} ; a_{3} a_{5}}\left(d_{1}, d_{2}\right)
$$

Where

$$
\begin{aligned}
K_{n_{1} m_{1}}^{n_{2} m_{2}} & =\sum_{k_{1}=0}^{n_{1}} \sum_{k_{2}=0}^{m_{1}} \sum_{k_{3}=0}^{n_{2}} \sum_{k_{4}=0}^{m_{2}} F_{k_{1}}\left(n_{1}\right) F_{k_{2}}\left(m_{1}\right) F_{k_{3}}\left(n_{2}\right) F_{k_{4}}\left(m_{2}\right) g_{k_{1} k_{2}}^{k_{3} k_{4}} i^{k_{1}+k_{2}+k_{3}+k_{4}}, \\
g_{k_{1} k_{2}}^{k_{3} k_{4}} & =\Gamma\left(\frac{1+k_{1}}{2}\right) \Gamma\left(\frac{1+k_{2}}{2}\right) \Gamma\left(\frac{1+k_{3}}{2}\right) \Gamma\left(\frac{1+k_{4}}{2}\right) \\
\times & {\left[1+(-1)^{k_{1}}\right]\left[1+(-1)^{k_{2}}\right]\left[1+(-1)^{k_{3}}\right]\left[1+(-1)^{k_{4}}\right] }
\end{aligned}
$$

and

$$
I_{n_{1} m_{1} ; a_{1} a_{2} a_{3}}^{n_{2} m_{3} ; a_{3} a_{5}}\left(d_{1}, d_{2}\right)=\frac{2^{n_{1}+m_{1}+n_{2}+m_{2}}}{(4 \pi)^{2}} \int_{-\infty}^{\infty} \int_{-\infty}^{\infty} a_{1}^{n_{1}-k_{1}} a_{2}^{m_{1}-k_{2}} a_{3}^{n_{2}-k_{3}} a_{4}^{m_{2}-k_{4}} e^{-\frac{1}{2}\left(a_{3}{ }^{2}+a_{4}{ }^{4}+a_{5}\right)} d x_{1} d x_{2} .
$$


Taking into account the multinomial expansion theorem in Eq. (16);

$$
(x+y+z)^{n}=\sum_{i=0}^{n} \sum_{j=0}^{n-i} F_{i}(n) F_{j}(n-i) x^{n-i-j} y^{j} z^{i}
$$

We obtain

$$
\begin{aligned}
I_{n_{1} m_{1} ; a_{1} a_{2} a_{3}}^{n_{2} m_{3} a_{3} a_{5}} & \left(d_{1}, d_{2}\right)= \\
S_{n_{1} m_{1} k_{1} k_{2}}^{n_{2} m_{2} k_{3} k_{4}} & \sum_{k_{5}=0}^{n_{2}-k_{3}} \sum_{k_{6}=0}^{n_{2}-k_{3}-k_{5}} \sum_{k_{7}=0}^{m_{2}-k_{4}} \sum_{k_{8}=0}^{m_{2}-k_{4}-k_{7}} F_{k_{5}}\left(n_{2}-k_{3}\right) F_{k_{6}}\left(n_{2}-k_{3}-k_{5}\right) F_{k_{7}}\left(m_{2}-k_{4}\right) F_{k_{8}}\left(m_{2}-k_{4}-k_{7}\right) \\
& \times\left(J_{11}\right)^{n_{2}-k_{3}-k_{5}-k_{6}}\left(J_{12}\right)^{k_{6}}\left(J_{21}\right)^{m_{2}-k_{4}-k_{7}-k_{8}}\left(J_{22}\right)^{k_{8}} d_{1}^{k_{5}} d_{2}^{k_{7}} Q_{c_{1} c_{3} c_{5} c_{7}}^{c_{2} c_{4} c_{5}}
\end{aligned}
$$

where

$$
\begin{aligned}
& S_{n_{1} m_{1} k_{1} k_{2}}^{n_{2} m_{2} k_{3} k_{4}}=\left(\sqrt{\alpha_{1}}\right)^{n_{1}-k_{1}}\left(\sqrt{\alpha_{2}}\right)^{m_{1}-k_{2}}\left(\sqrt{\beta_{1}}\right)^{n_{2}-k_{3}}\left(\sqrt{\beta_{2}}\right)^{m_{2}-k_{4}} \frac{1}{(4 \pi)^{2}} \prod_{i=1}^{2} 2^{n_{i}+m_{i}} \exp \left[-\frac{d_{i}^{2} \beta_{i}}{2}\right] \\
& Q_{c_{1} c_{3} c_{5} c_{7}}^{c_{2} c_{1} c_{6}}=\int_{-\infty}^{\infty} \int_{-\infty}^{\infty} x_{2}^{c_{2}} e^{-c_{4} x_{2}{ }^{2}-c_{6} x_{2}} x_{1}^{c_{1}} e^{-c_{3} x_{1}{ }^{2}-\left(c_{5}+c_{7} x_{2}\right) x_{1}} d x_{1} d x_{2} .
\end{aligned}
$$

In Eq. (20), the coefficients are as follows:

$$
\begin{aligned}
& c_{1}=n_{1}+n_{2}+m_{2}-k_{1}-k_{3}-k_{4}-k_{5}-k_{6}-k_{7}-k_{8} \\
& c_{2}=m_{1}+k_{6}+k_{8}-k_{2} \\
& c_{3}=\frac{1}{2} \sum_{i=1}^{2} \beta_{i} J_{i 1}^{2}+\alpha_{1} \\
& c_{4}=\frac{1}{2} \sum_{i=1}^{2} \beta_{i} J_{i 2}{ }^{2}+\alpha_{2} \\
& c_{5}=\sum_{i=1}^{2} d_{i} \beta_{i} J_{i 1} \\
& c_{6}=\sum_{i=1}^{2} d_{i} \beta_{i} J_{i 2} \\
& c_{7}=\sum_{i=1}^{2} \beta_{i} J_{i 1} J_{i 2}
\end{aligned}
$$


The solution of the integral in Eq. (20) is given as follows [30]:

$$
\int_{-\infty}^{\infty} y^{n} e^{-p y^{2}+2 q y} d y=n ! e^{q^{2} / p} \sqrt{\frac{\pi}{p}}\left(\frac{q}{p}\right)^{n} \sum_{k=0}^{E(n / 2)} \frac{1}{(n-2 k) ! k !}\left(\frac{p}{4 q^{2}}\right)^{k} \quad \text { for } \quad p>0
$$

where is

$$
E(n / 2)=\frac{n}{2}-\frac{1}{4}\left[1-(-1)^{n}\right]
$$

Taking into account Eq. (28) into Eq. (20), we can obtain the formulae

$$
\begin{aligned}
& Q_{c_{1} c_{3} c_{5} c_{7}}^{c_{2} c_{2} c_{6}}=\frac{c_{1} !}{2^{c_{1}}} \sqrt{\pi} \sum_{r=0}^{E\left(c_{1} / 2\right)} \frac{c_{3}^{r-c_{1}-1 / 2}(-1)^{c_{1}-2 r}}{\left(c_{1}-2 r\right) ! r !} \sum_{i=0}^{c_{1}-2 r} f_{i}\left(c_{1}-2 r\right) c_{5}^{c_{1}-2 r-i} c_{7} e^{{ }^{c_{5}}{ }^{2} / 4 c_{3}} \\
& \times\left(c_{2}+i\right) ! e^{b_{2}{ }^{2} / b_{1}} \sqrt{\frac{\pi}{b_{1}}}\left(\frac{b_{2}}{b_{1}}\right)^{c_{2}+i} \sum_{r^{\prime}=0}^{E\left(\left(c_{2}+i\right) / 2\right)} \frac{1}{\left(c_{2}+i-2 r^{\prime}\right) ! r^{\prime} !}\left(\frac{b_{1}}{4 b_{2}{ }^{2}}\right)^{r^{\prime}}
\end{aligned}
$$

where

$$
\begin{aligned}
& b_{1}=c_{4}-\frac{c_{7}^{2}}{4 \cdot c_{3}}, \\
& b_{2}=\frac{c_{5} \cdot c_{7}}{4 \cdot c_{3}}-\frac{c_{6}}{2} .
\end{aligned}
$$

The squares of the Franck-Condon (FC) integrals are the so-called FC factor, which is given by

$$
F C F=\left\langle n_{1} m_{1} \mid n_{2} m_{2}\right\rangle^{2}
$$

Depending on the temperature and the FCF, the spectral intensity $(I)$, is [26]:

$$
I=c \times F C F \times \exp \left(-\frac{E_{v}}{k_{B} T}\right)
$$

Where $c$ is a proportion constant, $E_{v}$ is the energy of the initial state, $k_{B}$ is the Boltzmann constant, and $T$ is the temperature [26]. As can be seen from Eqs. (14), (18) and (30), the final formula is expressed in terms of binomial coefficients $F_{m}(n)$. In order to avoid the calculation 
of factorials of integers in the formula of binomial coefficients, we use the following recurrence relation:

$F_{m}(n)=\left(\frac{n+1}{m}-1\right) F_{m-1}(n)$

The coefficients $F_{m}(n)$ are repeatedly needed in computations. In order to put these coefficients into or to get them back from the memory, the position of certain coefficients $F_{m}(n)$ is determined by the relation:

$F_{n m}=\frac{n(n+1)}{2}+m+1$

The suggested approach provides a considerable reduction of computational time of the twodimensional FCF [16, 31, 32].

For FCF calculations of $\mathrm{NO}_{2}$ molecule, vibration frequencies are $\left(\omega_{1}, \omega_{2}\right)=(1358,757) \mathrm{cm}^{-1}$, $\left(\omega_{1}^{\prime}, \omega_{2}^{\prime}\right)=(1461,739) \mathrm{cm}^{-1}[33]$. Using $\alpha_{i}=\omega_{i} / \hbar, \beta_{i}=\omega^{\prime} / \hbar$ equations, we can obtain the $\alpha_{i}$ and $\beta_{i}$ values in the atomic unit system as $\left(\alpha_{1}, \alpha_{2}\right)=\left(6.2 \times 10^{-3}, 3.5 \times 10^{-3}\right) a . u$, $\left(\beta_{1}, \beta_{2}\right)=\left(6.7 \times 10^{-3}, 3.4 \times 10^{-3}\right)$ a.u.

For $\mathrm{NO}_{2}$ molecule, the Duschinsky matrix components are given by [33],

$$
\begin{aligned}
& \left(\begin{array}{ll}
J_{11} & J_{12} \\
J_{21} & J_{22}
\end{array}\right)=\left(\begin{array}{cc}
0.938 & -0.346 \\
0.346 & 0.938
\end{array}\right) \\
& \left(\begin{array}{l}
d_{1} \\
d_{2}
\end{array}\right)=\left(\begin{array}{c}
-1.161 \\
2.146
\end{array}\right)
\end{aligned}
$$

Accordingly, the results obtained ${ }^{1}$ for ${ }^{2} B_{2}-{ }^{2} A_{1}$ band system of $\mathrm{NO}_{2}$ molecule were given in Tables 1-3.

\footnotetext{
${ }^{1}$ In FCF calculations of $\mathrm{NO}_{2}$ molecule for the values vibration frequency, the authors contacted with K.M. CHEN.
} 


\section{Results and Discussion}

In this study, we derived a new analytical formula for the two-dimensional FC factors on a harmonic oscillator basis. The binomial coefficients $F_{m}(n)$ are repeatedly needed in a computation of the two-dimensional FC factors. The algorithm for the storage of coefficients $F_{m}(n)$ suggested has important contributions in reducing the requirements for computer time on the calculations $[31,32,34]$. Therefore, the proposed formula helps us to calculate quickly the FC factor for the arbitrary values of $n_{1}, m_{1}, n_{2}, m_{2}$.

The obtained results are shown in Table 1 and Figs.1-4. Figures 1-4 show intensity alternations between even and odd vibrational quantum numbers $\left(n_{1}, m_{1}, n_{2}, m_{2}\right)$. The vibrational quantum numbers used in the calculations were completely arbitrarily chosen. Table 1 shows the FCF values for different quantum numbers.

All values in Tables 2 and 3 seem to be quite small. However, it is seen that the $(0,0),(0,2)$, $(2,0),(1,0),(1,1),(2,2)$ transitions in Table 1 are larger than the others. It also can be said that it is weak for the spectra of the $n_{2} \geq 3, m_{2} \geq 3$ states. In the same way, it is shown that the $(0,0),(0,1),(0,2),(0,4),(1,1),(2,0),(2,2),(0,4)$ transitions in Table 2 appear to be larger than the others. In the same way, the spectra of the $n_{1} \geq 3, m_{1} \geq 3$ states is weak.

Fig. 1 shows the $n_{1}$ variation of FCF for the $(0,0,0,0),(1,0,0,0), \ldots,(12,0,0,0)$ bands. From the Fig. 1, it seems that the FCFs with increased $\mathrm{n}_{1}$ for transitions are negligibly small, and this is almost identical in other figures.. Also, the small variations in figures are shown clearly. This implies that the FCF method used is very sensitive for obtaining reliable results for the studied molecules. We can also say that the FCF values are becoming more and more small values when $\mathrm{m}_{1}, \mathrm{n}_{2}$ and $\mathrm{m}_{2}$ receive the different values from zero. 
The $n_{1}$ variation of FCF for the $m_{1}=n_{2}=m_{2}=1$ is displayed in Fig. 2. Similarly in Fig. 1 , the calculated FCF values decrease with increased $n_{1}$. At low $n_{1}$ values, the FCF values have bigger values. It is shown from Fig. 2 that the small changes in FCF values is more apparent with the variation of $m_{1}, n_{2}$ and $m_{2}$ values. All of figures show clearly that the other quantum numbers with the quantum number $n_{1}$ has a very significant role in calculation of FCF.

Fig. 3 shows the FCF calculated depending on $n_{1}$ for the $(0,1,0,2),(1,1,0,2), \ldots,(12,1,0,2)$ vibrations states of $\mathrm{NO}_{2}$ molecule. Note that the FCFs have smaller values with the values of $m_{1}=1, n_{2}=0$ and $m_{2}=2$. In $n_{1}=1$, the FCF has the maximum value as in Fig. 2.

The variation of FCF with $n_{1}$ is shown Fig. 4. In Fig. 4, it is seems that there are peak intensity compared to the other Figures. However, the FCF values are extremely small. From all of Figures, it seems that the FCF values decreases with increased mixing of $m_{1}, n_{2}$ and $m_{2}$ values. The maximum value of the FCF is in $n_{1}=5$.

When we look at whole Figures, the peaks obtained for the statement of $m_{1}=n_{2}=m_{2}=0$ of the quantum numbers are weak and, therefore the values obtained for the other conditions could provide more sensitive information about the FCF of molecules.

\section{Conclusion}

A new analytical expression for the calculation of generalized FC factors of 2D harmonic oscillators including the Duschinsky effect has been developed by the use of binomial coefficients and Hermite polynomials. Typically, the computation of the 2D FC factors places a heavy burden on the CPU time as well as the memory requirements, especially with high quantum numbers. The CPU time of the presented method is less. Therefore, the obtained formulas are computationally efficient for practical calculations. The presented method in this 
report is completely general can be useful for accurately calculating the intensity distribution in the photoelectron spectrum and nonradiative processes of molecules.

\section{Acknowledgement:}

The authors wish to thank Prof. Dr. K.M. CHEN for valuable contributions and comments in the work.

\section{References}

[1] H. Kupka and P.H. Cribb, 1986, J. Chemical Physics, 85, 1303.

[2] K. Chen and C. Pei, 1990, J. Molecular Spectroscopy, 140, 401.

[3] K. Chen and C. Pei, 1990, Chemical Physics Letters, 165, 523.

[4] F. Iachello and S. Oss, 1991, Phys. Rev. Lett., 66, 2976.

[5] M. Lee and M.S. Kim, 2003, J. Chemical Physics, 119, 10.

[6] M. Malagoli, V. Coropceanu, D.A. da Silva Filho and J.L. Bre'das, 2004, J. Chemical Physics, 120,16.

[7] J. Liang and H.J. Li, 2005, Molecular Physics, 103, 3337.

[8] J.M. Luis, M. Torrent-Sucarrat, M. Sola, D.M. Bishop, B. Kirtman, 2005, The Journal of Chemical Physics 122, 18410

[9] J.M. Luis, B. Kirtman, O. Christiansen, 2006, J. Chem. Phys. 125, 154114.

[10] I.I. Guseinov, B.A. Mamedov, A.S. Ekenoğlu, 2006, Z. Naturforsch., 61,141.

[11] I.I. Guseinov, B.A. Mamedov, A.S. Ekenoğlu, 2006, Comput. Phys. Commun., 175, 226.

[12] J. Liang, H. Zheng, X. Zhang, R. Li and Z. Cui, 2007, Molecular Physics, 105, 1903.

[13] J. Liang, F. Cui, R. Wang and Z. Cui, 2009, Molecular Physics, 107, 2601

[14] J. Liang, F. Cui, R. Wang, W. Huang, Z. Cui, 2013, J. Molecular Spectroscopy, 286, 12

[15] H.M. Huang and J. Liang, 2011, J. Atomic Molecular Sciences, 2, 179

[16] B.A. Mamedov, H. Koç, N. Sünel, 2013, J. Molecular Structure, 1048, 301.

[17] F. Duschinsky, 1937, ActaPhysicochimica U.R.S.S., 7, 551.

[18] J. Franck, E.G. Dymond, 1925, Trans. Faraday Soc., 21, 536.

[19] E. Condon, 1928, Physical Review, 32, 858.

[20] V.E. Bondybey, 1984, Chemical Physics Letters, 109, 5.

[21] M. Wagner, 1959, Z. Naturforschg. Teil A, 14, 18

[22] P. Sriramachandran, R. Shanmugavel, 2011, New Astronomy, 16, 110

[23] G.M. Sando, K.G. Spears and J.T. Hupp, 2001, J. Physical Chemistry A, 105, 5317. 
[24] B.A. Mamedov, 2013, Int. J. Quantum Chemistry, 113, 1372

[25] J. Subbi, 1988, Chemical Physics, 122, 157

[26] C.L. Lee, S.H. Yang, S.Y. Kuo, J.L. Chang, 2009, J. Molecular Spectroscopy, 256, 279.

[27] M. Dierksen and S. Grimme, 2005, J. Chemical Physics, 122, 244101.

[28] R. Berger, C. Fischer and M. Klessinger, 1998, J. Phys. Chem. A, 102, 7157-7167

[29] V. Rodriguez-Garcia, K. Yagi, K. Hirao, S. Iwata, S. Hirata, 2006, The Journal Of Chemical Physics 125, 014109

[30] I.S. Gradshteyn, I.M. Ryzhik, 1980, Table of Integrals, Series and Products; Academic: New York

[31] I.I. Guseinov, 1997, Journal of Molecular Structure (Theochem), 1997, 417,117.

[32] I.I. Guseinov, B.A. Mamedov, 2005, Applied Mathematics and Computation, 161, 285.

[33] K. Chen and C. Pei, 1990, Chemical Physics, 144, 383-389.

[34] B.A. Mamedov, 2004, Chinese Journal of Physics, 42, 176 


\section{FIGURES CAPTIONS}

Figure 1. The $n_{1}$ variation of FCF for ${ }^{2} B_{2}-{ }^{2} A_{1}$ band system and the $(0,0,0,0),(1,0,0,0), \ldots$, $(12,0,0,0)$ vibrational quantum numbers of $\mathrm{NO}_{2}$ molecule bands.

Figure 2. The $n_{1}$ variation of FCF for ${ }^{2} B_{2}-{ }^{2} A_{1}$ band system and the $(0,1,1,1),(1,1,1,1), \ldots$, $(9,1,1,1)$ vibrational quantum numbers of $\mathrm{NO}_{2}$ molecule bands.

Figure 3. The $n_{1}$ variation of FCF for ${ }^{2} B_{2}-{ }^{2} A_{1}$ band system and the $(0,1,0,2),(1,1,0,2), \ldots$, $(9,1,0,2)$ vibrational quantum numbers of $\mathrm{NO}_{2}$ molecule bands.

Figure 4. The $n_{1}$ variation of FCF for ${ }^{2} B_{2}-{ }^{2} A_{1}$ band system in the statement of the different vibrational quantum numbers of $\mathrm{NO}_{2}$ molecule bands.

\section{TABLE CAPTIONS}

Table 1. Values of Franck-Condon integrals for different quantum numbers

Table 2. Values of Franck-Condon Factor of the different quantum numbers for ${ }^{2} B_{2}-{ }^{2} A_{1}$ band system of $\mathrm{NO}_{2}$ molecule bands $\left(n_{1}=0, m_{1}=0\right)$.

Table 3. Values of Franck-Condon Factor of the different quantum numbers for ${ }^{2} B_{2}-{ }^{2} A_{1}$ band system of $\mathrm{NO}_{2}$ molecule bands $\left(n_{2}=0, m_{2}=0\right)$. 


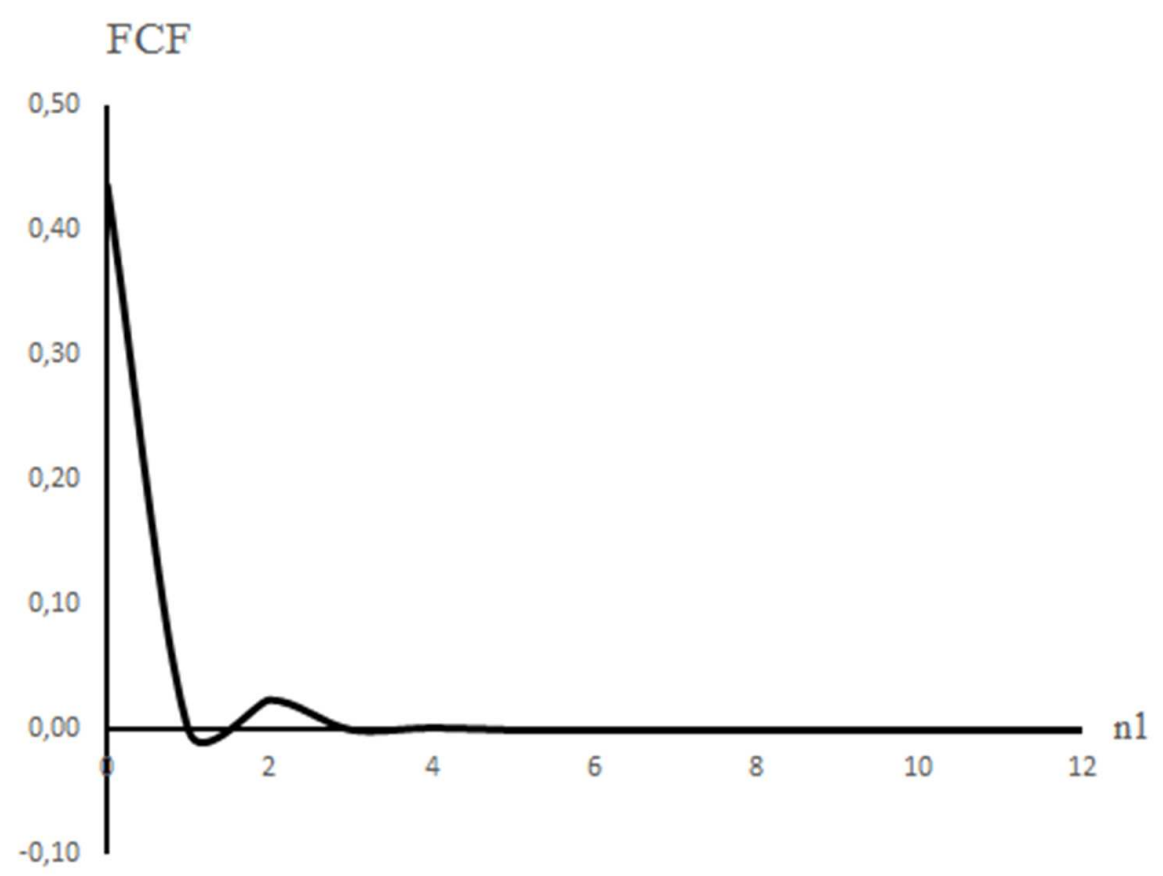

Fig. 1.

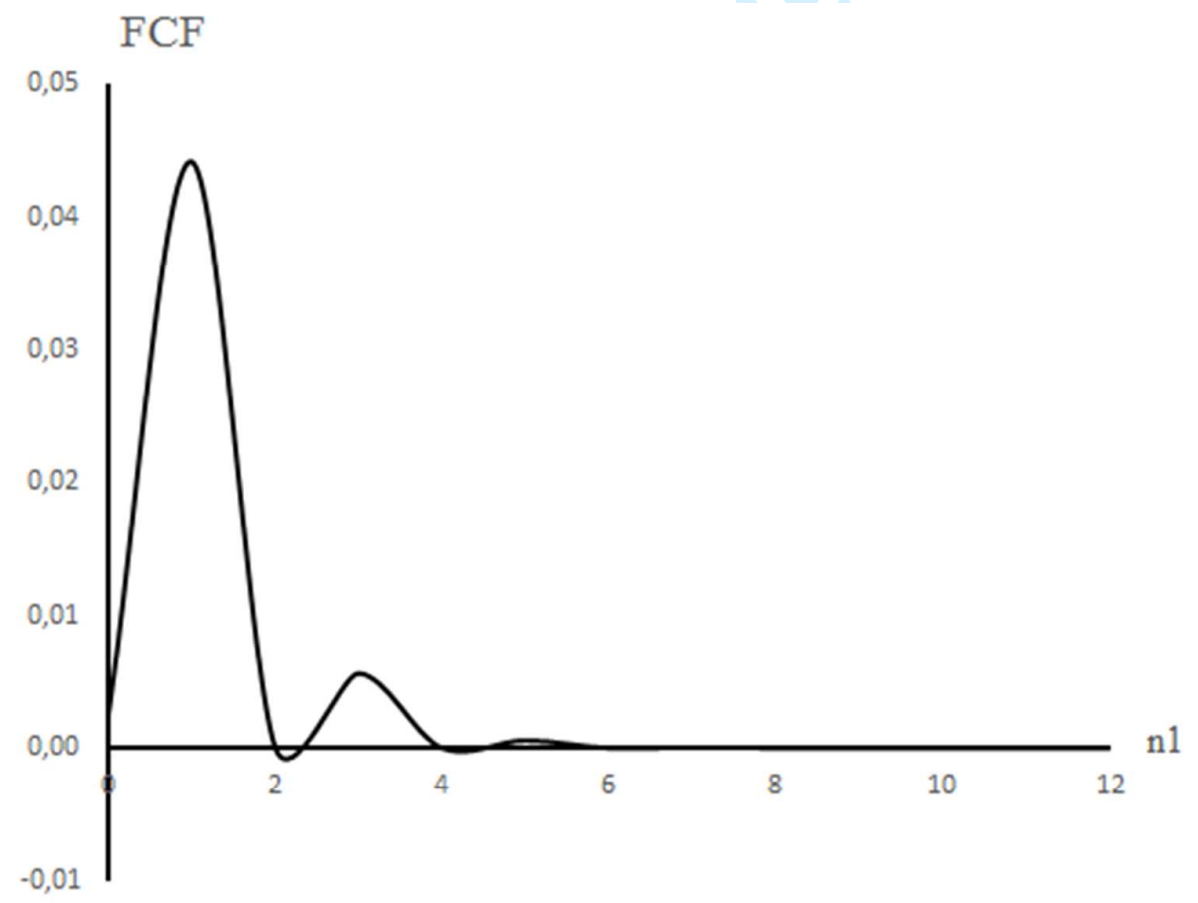

Fig. 2. 


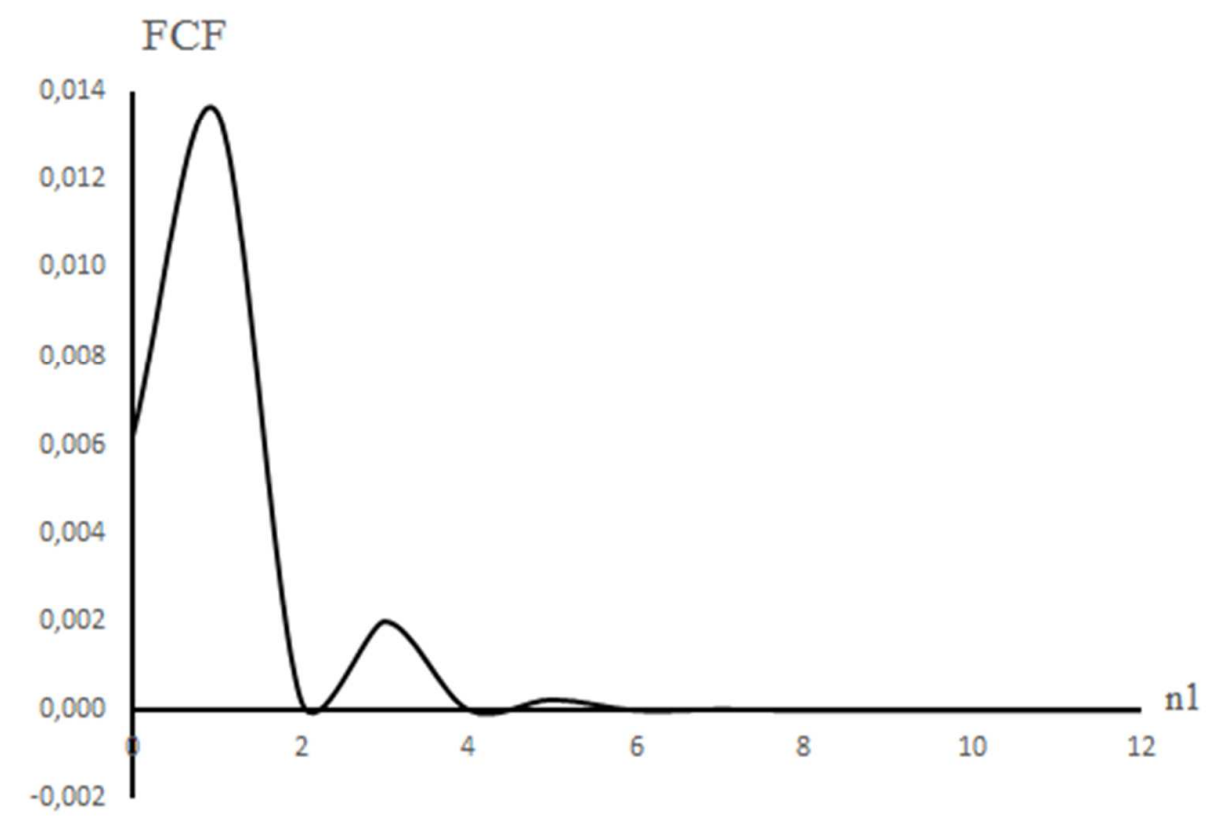

Fig. 3.

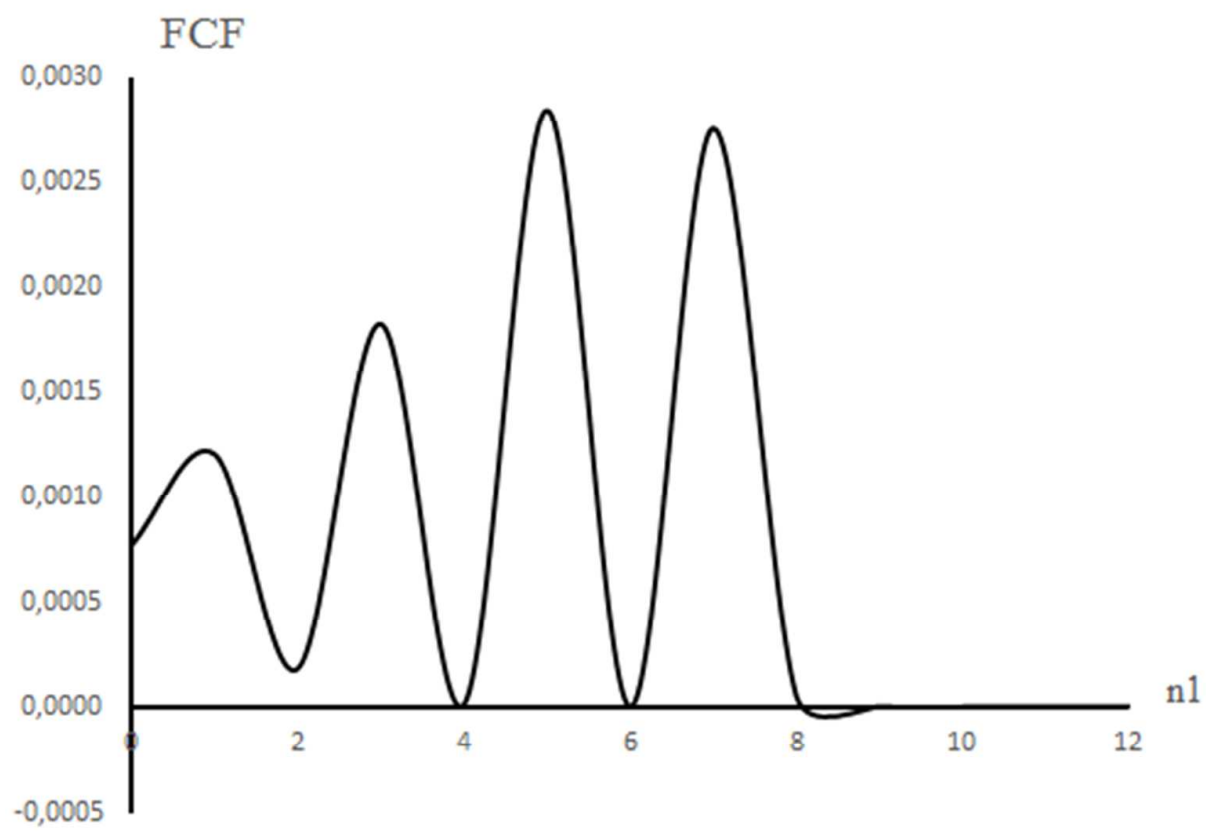

Fig. 4. 


\section{Table 1.}

\begin{tabular}{|l|l|l|l|l|}
\hline$n_{1}$ & $m_{1}$ & $n_{2}$ & $m_{2}$ & $\begin{array}{c}F C F=\left\langle n_{1} m_{1} \mid n_{2} m_{2}\right\rangle^{2} \\
E q .(32)\end{array}$ \\
\hline 0 & 0 & 0 & 0 & $4.362281462451970744 \mathrm{E}-01$ \\
\hline 1 & 0 & 1 & 0 & $1.702235961823670861 \mathrm{E}-01$ \\
\hline 0 & 1 & 0 & 1 & $1.609353834400126059 \mathrm{E}-01$ \\
\hline 1 & 1 & 0 & 0 & $1.007681237084941159 \mathrm{E}-03$ \\
\hline 1 & 1 & 0 & 1 & $7.083181982308392447 \mathrm{E}-06$ \\
\hline 2 & 1 & 0 & 1 & $7.141383464892485746 \mathrm{E}-03$ \\
\hline 2 & 0 & 1 & 0 & $6.474268889715763865 \mathrm{E}-04$ \\
\hline 1 & 0 & 2 & 1 & $6.437168015380755421 \mathrm{E}-03$ \\
\hline 2 & 2 & 1 & 2 & $1.206338385233231894 \mathrm{E}-05$ \\
\hline 1 & 3 & 0 & 3 & $1.557593874636091381 \mathrm{E}-04$ \\
\hline 1 & 3 & 1 & 1 & $1.007025178170728003 \mathrm{E}-02$ \\
\hline 1 & 4 & 0 & 2 & $4.626535058800947712 \mathrm{E}-07$ \\
\hline 2 & 7 & 0 & 4 & $8.693240997764169224 \mathrm{E}-05$ \\
\hline 2 & 9 & 0 & 1 & $4.628292897770967410 \mathrm{E}-06$ \\
\hline 4 & 3 & 0 & 0 & $3.056970602983083767 \mathrm{E}-06$ \\
\hline 6 & 4 & 0 & 0 & $1.480495159093913278 \mathrm{E}-06$ \\
\hline 7 & 7 & 0 & 0 & $1.326823018523905874 \mathrm{E}-08$ \\
\hline 11 & 9 & 0 & 0 & $3.451784178563368181 \mathrm{E}-11$ \\
\hline 3 & 1 & 3 & 2 & $5.140529248241911362 \mathrm{E}-05$ \\
\hline 4 & 2 & 1 & 3 & $1.514930209216614861 \mathrm{E}-03$ \\
\hline 5 & 4 & 2 & 4 & $4.552541318064996184 \mathrm{E}-06$ \\
\hline 5 & 6 & 3 & 1 & $4.816249320714024051 \mathrm{E}-05$ \\
\hline 7 & 6 & 4 & 2 & $3.343636618699123100 \mathrm{E}-05$ \\
\hline 8 & 7 & 0 & 1 & $2.495756934213322324 \mathrm{E}-08$ \\
\hline 9 & 1 & 3 & 0 & $2.462945267283240271 \mathrm{E}-05$ \\
\hline 10 & 0 & 0 & 5 & $5.592110274511242862 \mathrm{E}-08$ \\
\hline 11 & 0 & 10 & 0 & $4.371885472733922696 \mathrm{E}-04$ \\
\hline 12 & 1 & 5 & 0 & $2.338302252642711406 \mathrm{E}-04$ \\
\hline
\end{tabular}




\section{Table 2.}

\begin{tabular}{|l|l|l|l|l|l|l|l|}
\hline$n_{2} / m_{2}$ & 0 & 1 & 2 & 3 & 4 & 5 & Sum \\
\hline 0 & 0.4363 & 0.0059 & 0.0285 & 0.0012 & 0.0028 & 0.0002 & 0.4749 \\
\hline 1 & 0.0026 & 0.0037 & 0.00002 & 0.0007 & 0.000003 & 0.0001 & 0.007123 \\
\hline 2 & 0.0144 & 0.00006 & 0.00132 & 0.00002 & 0.0002 & 0.0000005 & 0.016 \\
\hline 3 & 0.00027 & 0.00037 & 0.0000004 & 0.00008 & 0.00000005 & 0.000015 & 0.00074 \\
\hline 4 & 0.0007 & 0.00000001 & 0.00009 & 0.00000003 & 0.00001 & 0.00000002 & 0.0008 \\
\hline 5 & 0.000023 & 0.0000306 & 0.00000006 & 0.0000008 & 0.0000000001 & 0.0000002 & 0.000055 \\
\hline Sum & 0.4543 & 0.01006 & 0.02993 & 0.002 & 0.003 & 0.0003 & \\
\cline { 1 - 4 } & & & & & & &
\end{tabular}

Table 3.

\begin{tabular}{|l|l|l|l|l|l|l|l|}
\hline$n_{1} / m_{1}$ & 0 & 1 & 2 & 3 & 4 & 5 & Sum \\
\hline 0 & 0.4363 & 0.0023 & 0.0256 & 0.00041 & 0.002252 & 0.0000611 & 0.466923 \\
\hline 1 & 0.0002 & 0.00101 & 0.00005 & 0.0002 & 0.00000096 & 0.000026 & 0.001487 \\
\hline 2 & 0.0241 & 0.0002 & 0.002 & 0.00003 & 0.00015 & 0.00000047 & 0.026481 \\
\hline 3 & 0.00004 & 0.00017 & 0.0000009 & 0.00003 & 0.0000002 & 0.0000005 & 0.000242 \\
\hline 4 & 0.0020 & 0.000015 & 0.00014 & 0.0000003 & 0.000014 & 0.00000005 & 0.002169 \\
\hline 5 & 0.0000003 & 0.000023 & 0.00000012 & 0.00000043 & 0.00000003 & 0.00000007 & 0.000024 \\
\hline Sum & 0.46264 & 0.003718 & 0.027791 & 0.000671 & 0.0024172 & 0.000882 & \\
\cline { 1 - 5 } & & & & & & & \\
\cline { 1 - 3 } & & & & & &
\end{tabular}

